\title{
The difficulty to diagnose and treat a rare disease: response to midostaurin in a case of aggressive systemic mastocytosis conditioning bulky lymphadenopathy
}

Mariarita Sciumé ${ }^{1}$, Fabio Serpenti ${ }^{2}$, Simona Muratori ${ }^{3}$, Valerio Pravettoni ${ }^{4}$, Fabio Massimo Ulivieri $^{5}$, Giorgio Alberto Croci $^{6}$, Anna Chiara Migliorini ${ }^{7}$, Andrea Esposito ${ }^{8}$, Maria Cecilia Goldaniga ${ }^{1}$, Giorgia Natascia Saporiti ${ }^{1}$, Giulia Galassi ${ }^{1}$, Luca Baldini ${ }^{1}$, Francesco Onida $^{1}$, and Federica Irene Grifoni ${ }^{9}$

${ }^{1}$ Hematology Unit, Fondazione IRCCS Ca' Granda Ospedale Maggiore Policlinico, Milan, Italy

${ }^{2}$ Università degli Studi di Milano, Milan, Italy

${ }^{3}$ Dermatology Unit, Department of Physiopathology and Transplantation, Fondazione

IRCCS Ca' Granda Ospedale Maggiore Policlinico, Università di Milano

${ }^{4}$ U.O.C. General Medicine - Immunology and Allergology, Fondazione Ca' Granda IRCCS, Ospedale Maggiore Policlinico, Università di Milano

${ }^{5}$ Nuclear Medicine, Bone Metabolic Unit, Fondazione IRCCS Ca' Granda Ospedale Maggiore Policlinico, Università di Milano

${ }^{6}$ Division of Pathology, Department of Pathophysiology and Transplantation, Fondazione IRCCS Ca' Granda Ospedale Maggiore Policlinico, Milan, Italy

${ }^{7}$ Laboratorio di ematologia, Servizio di immunoematologia e trasfusionale, Fondazione Ca' Granda IRCCS Ospedale Maggiore Policlinico

${ }^{8}$ Division of Radiology, Fondazione IRCCS Ca' Granda Ospedale Maggiore Policlinico, Milan, Italy

${ }^{9}$ Hematology Unit, Fondazione Ca' Granda IRCCS, Ospedale Maggiore Policlinico, Università di Milano

June 12, 2020

\begin{abstract}
Systemic mastocytosis (SM) can be extremely heterogeneous and its treatment should be highly individualized. We reported a description of clinical management of an aggressive SM characterized at diagnosis by multiple organ involvement. The patient underwent to different lines of treatment, from standard chemotherapy to the novel tyrosine kinase inhibitor midostaurin.
\end{abstract}

\section{Introduction}

Systemic mastocytosis (SM) results from a clonal proliferation of abnormal mast cells (MCs) in extracutaneous organs. It could be divided into indolent SM (ISM), smoldering SM (SSM), SM with an associated hematologic (non-MC lineage) neoplasm (SM-AHNMD), aggressive SM (ASM), and mast cell leukemia (MCL). [1-3]

Clinical features of SM could be related to histamine release or to uncontrolled growth and infiltration of 
clonal MCs in different organs. [2-5] The latter clinical features are divided into B findings ( $>30 \% \mathrm{MC}$ infiltration on BM biopsy and serum total tryptase level $>200 \mathrm{ng} / \mathrm{mL}$, hepatomegaly with normal liver function, palpable splenomegaly without hypersplenism, and/or lymphadenopathy, signs of dysplasia or myeloproliferation in non-MC lineage, but not diagnostic of an associated hematological neoplasm) or $\mathrm{C}$ findings (organ dysfunction due to mast cell infiltration). [2-4] Diagnosis of ISM is established with $<2$ B findings and no $\mathrm{C}$ findings; when $>2 \mathrm{~B}$ findings but no $\mathrm{C}$ findings are present, the diagnosis is SSM, and when one or more $\mathrm{C}$ findings are detected ASM can be diagnosed. [2-4]

The treatment of adult SM should be highly individualized; while the backbone of therapy in ISM and SSM is symptom management, for aggressive and leukemic forms cytoreductive treatment is indicated. [3,4] Historically, cytoreductive agents include interferon- $\alpha$ and cladribine. [3,4,6,7] Allogeneic stem cell transplant could be considered in SM-AHNMD when the associated hematologic neoplasm has an indication to transplantation and in ASM patients with relapsed/refractory disease or in those with acute MCL. [8]

With the advent of the tyrosine kinase inhibitors (TKIs) era, many efforts have been made to find proper inhibitor of SM c-KIT -driver mutation. Midostaurin has been shown to induce major clinical responses with improvement or disappearance of one or more C-findings, a decrease in the MC burden, organomegaly and mediator-related symptoms. [9]

Hereby, we report an extended description of a real-life management of an ASM patient conditioning bulky lymphadenopathy who has undergone to multiple lines of treatment.

All procedures were in accordance with the ethical standards of the responsible committee on human experimentation (institutional and national) and with the Helsinki Declaration of 1975, as revised in 2013.

\section{Case report}

In 30-year-old Caucasian man was referred to our Center for a ten-year history of maculopapular rash, intractable itching, flushing and a six-month history of diarrhea (3-4 bowel movements per day with loose stools associated to a $<10 \%$ weight loss). The medical history was otherwise unremarkable, except for a non-steroidal anti-inflammatory drug-induced anaphylaxis.

Complete blood count, common coagulation, liver and renal function tests were in a normal range. A computed tomography (CT) scan showed diffuse lymphadenopathy (up to ) and mild hepatosplenomegaly. The screening serum tryptase level was $209 \mathrm{ng} / \mathrm{ml}$ (normal level $<5 \mathrm{ng} / \mathrm{ml}$ ). Gastric, duodenal, distal ileal and colonic biopsies revealed a diffuse mucosal mast cell infiltration, with abnormal expression of CD2 and CD25, compatible with SM diagnosis. An abdominal lymph node biopsy also confirmed the SM diagnosis and excluded a lymphoproliferative disease. At bone marrow biopsy multifocal clusters of spindling mast cells CD25+ were equal to $70 \%$ of total cellularity. Aspirate smears showed $<5 \%$ of mast cells and a direct sequencing of the KITgene was positive for the D $816 \mathrm{~V}$ mutation. A total skeleton X-ray was negative for a bone lytic or sclerotic involvement, while osteoporosis was diagnosed on the basis of reduced bone mineral density at dual-energy X-ray absorptiometry scan for which calcium/vitamin D supplements were started. Since the patient presented only B-findings, diagnosis of SSM was made and he was not considered a candidate for a cytoreductive treatment. The patient was instructed to avoid triggers of MC degranulation and therapy with H1-H2 histamine receptor blockers; sodium cromolyn was started with a symptomatic purpose for itching and diarrhea obtaining a good control.

During the follow-up, patient's general conditions slowly worsened with the appearance of a pathological weight loss $(>10 \%)$, a more severe diarrhea and a progressive enlargement of abdominal lymph nodes, liver and spleen. In April 2017 confluent abdominal lymphoadenopathy had a maximum diameter of 6 $\mathrm{cm}$, conditioning appearance of ileal dislocation and ascites due to SM liver involvement (histologically confirmed). Liver and spleen had a diameter of 24 and $17 \mathrm{~cm}$, while bone marrow involvement remained unchanged as for biopsy and morphology. Blood count was normal and serum tryptase level raised to 332 $\mathrm{ng} / \mathrm{ml}$. We concluded for aggressive evolution of SM. First line cytoreductive therapy was started in April 2017 with subcutaneous cladribine $0,14 \mathrm{mg} / \mathrm{Kg}$ per day for 5 days per cycle. A total of 5 cycles every 6 weeks 
were administered. Therapy was overall well tolerated and no grade $3 / 4$ hematologic toxicities were observed. By the end of the fifth cycle the disease had not yet reached a response with persistence of symptoms, $60 \%$ of $\mathrm{MC}$ involvement at bone marrow biopsy, and stable lymphadenopathy and hepatosplenomegaly. Serum tryptase level was $173 \mathrm{ng} / \mathrm{ml}$.

From December 2017 midostaurin was obtained through an expanded access use and started at a dosage of $25 \mathrm{mg}$ twice daily, gradually increased to $100 \mathrm{mg}$ every 12 hours.

Rapidly after starting midostaurin, the patient experienced a considerable improvement of general condition, clinical symptoms and body weight. After 3 months of midostaurin therapy, a bone marrow biopsy highlighted the reduction of MC infiltration to 30-40\%, lymphadenopathy decreased to a diameter of $27 \mathrm{~mm}$, hepatic and splenic diameter were 22 and $13 \mathrm{~cm}$. Serum tryptase was $26 \mathrm{ng} / \mathrm{ml}$. Unfortunately, the CT scan allowed to incidentally diagnose a subclinical pulmonary embolism of a segmental artery in the superior left lobe. Pulmonary embolism is reported among adverse events during midostaurin treatment, therefore we stopped the TKI treatment and start anticoagulant therapy. Midostaurin was safely resumed one month later; evaluation at 6 month showed only $10 \%$ of $\mathrm{MC}$ in the bone marrow biopsy and further reduction of lymphadenopathy to a maximum diameter of $22 \mathrm{~mm}$. Figure 1 and $\mathbf{2}$ respectively show the reduction of bone marrow MCs infiltrate and organomegaly over time.

Today the patient is still on midostaurin $100 \mathrm{mg}$ bid, completely asymptomatic and with good tolerance to the therapy. A human leukocyte antigen typing was performed, but allogeneic stem cell transplant isn't currently taken consideration. At the last assessment (February 2020) maximum diameter of abdominal lymphadenopathy and spleen were 1.5 and $13 \mathrm{~cm}$, serum tryptase level was $27 \mathrm{ng} / \mathrm{ml}$ and bone marrow MCs have stabilized on $10 \%$ of the global cellularity. The response was classified as partial according to Gotlib et al. [11]

For the clinical management of a SM patient the most relevant questions are whether the patient has or develops advanced SM and what treatment can be applied. Although the risk is relatively low, patients with ISM may progress to ASM, SM-AHNMD or MCL. [1-5]

Here, we report the gradual evolution of a SSM case in ASM, conditioning hepatomegaly with impairment of liver function and ascites, splenomegaly and abdominal lymphoadenopathy with ileal dislocation.

According to the current guidelines, ISM therapy is generally focused on symptom relief. [3,4] All patients should be advised to avoid any potential triggers (food, drinks, drugs) that may provoke MCs activation. High risk patients are advised to carry epinephrine self-injector-pens. Treatment options include histamine H1/H2-blockers, sodium cromolyn, leukotriene inhibitors, topical agents, aspirin, ketotifen, omalizumab, corticosteroids and MC cytoreductive therapy only in severe/refractory cases. [3,4] In our case, during the smoldering phase, the patient received H1-H2 histamine receptor blockers and sodium cromolyn for itching and diarrhea obtaining a good control.

When an aggressive phase was diagnosed, considering the burden of the disease and the absence of comorbidities, we decided to offer a cytoreductive treatment with cladribine. MC reducing therapy is indicated in advanced SM to prevent further organ damage, extend survival, often at the expense of significant side effects. [6,7] Cladribine was one of the first agent used for symptom relief and rapid MC debulking, with a demonstrated therapeutic activity in all SM subtypes and an overall response rate (ORR) of 50-60\%. [6,7] Myelosuppression and lymphopenia are the most frequent adverse events. [6,7] In our case cladribine was well tolerated without grade $3 / 4$ toxicities. With the advent of KIT -targeted TKIs, the use of cytoreductive therapy has declined. [3,4] The multikinase inhibitor midostaurin is currently approved for patients with advanced SM. In this population it demonstrated a $60 \%$ ORR and a good tolerability with few reported adverse events apart from hematological toxicity, nausea, vomit and diarrhea. [8]

Our patient received midostaurin after failure of first-line therapy with cladribine. Midostaurin lead to a significant improvement of organomegaly, liver function and body weight; moreover a substantial reduction in MC burden and mediator-related symptoms were observed, translating into a significant amelioration of 
patient's life quality. This treatment is still ongoing, with almost 2 years of therapy, allowing to obtain and keep a partial response.

In conclusion, mastocytosis is a rare disease with a complex biology and an unpredictable clinical course. The diagnosis and management of MC neoplasms is an emerging challenge and requires vast knowledge, a multidisciplinary diagnostic approach and personalized treatment strategy.

Disclosure Statement : FIG received compensation by Novartis as expert testimony and as a member of advisory board. The remaining authors declare no conflict of interest.

Author Contribution: MS, FS, MCG, GNS, GG, LB, FO and FIG provided the patient data regarding the hematological disease; MS, FS and FIG wrote the manuscript; SM, VP and FMU provided patient data for what concerns dermatological, allergological and rheumatological aspects; GAC performed the histological examinations of bone marrow, provided iconographic materials, interpreted these data and integrated them in the manuscript; ACM revised patient peripheral blood smears; AE provided radiological iconographic material. All authors critically read and approved the final manuscript.

\section{References}

Arber DA, Orazi A, Hasserjian R, et al. The 2016 revision to the World Health Organization classification of myeloid neoplasms and acute leukemia. Blood 2016;127(20):2391-2405.

2 Horny HP, Metcalfe DD, Akin C, et al. Mastocytosis. In: Swerdlow SH et al., eds. WHO Classification of Tumors of Hematopoietic and Lymphoid Tissues. Lyon, France: International Agency for Research and Cancer (IARC); 2017:62-69.

3 Pardanani A. Systemic mastocytosis in adults: 2019 update on diagnosis, risk stratification and management. Am J Hematol 2019;94(3):363-377.

4 Scherber RM, Borate U. How we diagnose and treat systemic mastocytosis in adults. Br J Haematol 2018;180(1):11-23.

5 Lim KH, Tefferi A, Lasho TL, et al. Systemic mastocytosis in 342 consecutive adults: survival studies and prognostic factors. Blood 2009; 113:5727-5736.

6 Lim KH, Pardanani A, Butterfield JH, Li CY, Tefferi A. Cytoreductive therapy in 108 adults with systemic mastocytosis: outcome analysis and response prediction during treatment with interferon-alpha, hydroxyurea, imatinib mesylate or 2-chlorodeoxyadenosine. Am J Hematol 2009;84:790-794.

7 Barete S, Lortholary O, Damaj G, et al. Long-term efficacy and safety of cladribine (2-CdA) in adult patients with mastocytosis. Blood 2015;126:1009-1016.

8 Ustun C, Gotlib J, Popat U, et al. Consensus opinion on allogeneic hematopoietic cell transplantation in advanced systemic mastocytosis. Biol Blood Marrow Transplant 2016;22:1348-1356.

9 Chandesris MO, Damaj G, Canioni D, et al. Midostaurin in advanced systemic mastocytosis. N Engl J Med 2016;374(26):2605-2607.

10 Escribano L, Alvarez-Twose I, Sánchez-Muñoz L, et al. Prognosis in adult indolent systemic mastocytosis: A long-term study of the Spanish Network on Mastocytosis in a series of 145 patients. J Allergy Clin Immunol $2009 ; 124,514-521$.

11 Gotlib J, Pardanani A, Akin C, et al. International Working Group-Myeloproliferative Neoplasms Research and Treatment (IWG-MRT) \& European Competence Network on Mastocytosis (ECNM) consensus response criteria in advanced systemic mastocytosis. Blood 2013; 121(13):2393-401. 

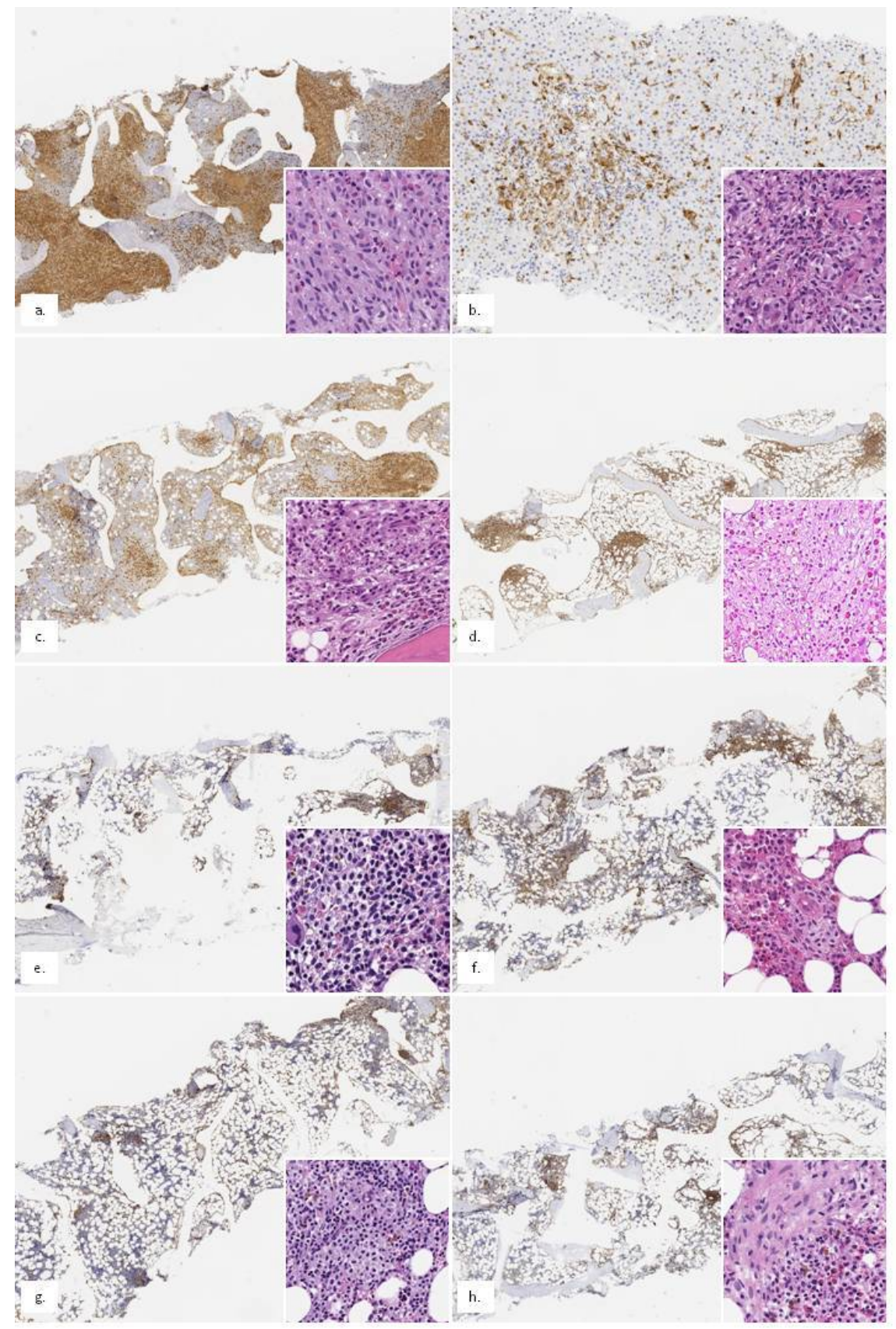

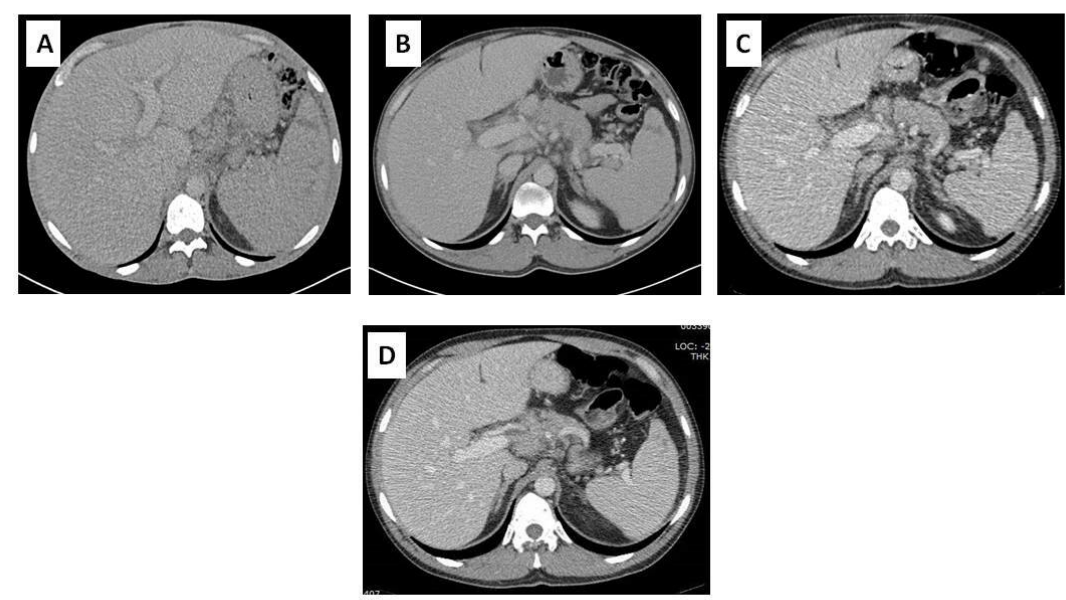